\title{
Reversibility of the inhibitory effect of salmon calcitonin on bone resorption in rats
}

\author{
Ugo E Pazzaglia', Giovanni Zatti', Paolo Rolla ${ }^{1}$ and Amalia Di Nucci ${ }^{2}$
}

Inhibition of osteoclastic bone resorption has been induced in growing rats with high doses of salmon calcitonin. This effect was evaluated by measuring the perichondrial ring height of the proximal tibial metaphysis. The aim was to assess whether osteoclastic activity resumed after a period of inhibition with high doses of calcitonin. 20 male Sprague-Dawley rats were treated for 21 days with 100 units/kg/day of salmon calcitonin subcutaneously and killed after 0-60 days, together with non-treated controls at 0 and 60 days.

Arrest of metaphyseal modeling and increased height of the perichondrial ring at the end of the period of therapy ( $P$ 0.002 versus controls) were observed. Recovery of bone resorption was evident 20 and 40 days after withdrawal of calcitonin.

'Clinica Ortopedica, II Facolta' di Medicina e Chirurgia dell'Universita' di Pavia Ospedale F. Del Ponte, I-21100 Varese, Italy, 2Istituto di Farmacologia II, Universita' di Pavia. Tel +39-332 282 682. Fax -332 288956

Submitted 93-04-03. Accepted 93-09-10

The capacity of calcitonin to inhibit bone resorption has been demonstrated in vitro (Chambers et al. 1985 , Reynolds and Dingle 1970, Stewart and Stern 1987) but not in animals with physiological doses of the hormone (Russel et al. 1973). High doses of salmon calcitonin were found to influence the morphology of the growth plate and metaphysis of growing rats: increased height of the perichondrial ring, persistence of cartilage intercolumnar septa in the outer part of the growth plate and failure of metaphyseal conization were observed. These changes can be explained by the inhibitory effect of the hormone on osteoclasts and the model employed can provide quantitative assessment of bone resorption inhibition (Pazzaglia et al. 1993). From these results arises the question of the reversibility of calcitonin action on osteoclasts. The aim of this study was to assess whether and at what time osteoclastic activity resumed after a period of inhibition with high doses of calcitonin.

\section{Material and methods}

20 male Sprague-Dawley rats (Stefano Morini, S. Polo d'Enza, Reggio Emilia, Italy), average weight $150 \mathrm{~g}$, were injected for 21 days with 100 units $/ \mathrm{kg} / \mathrm{day}$ of salmon calcitonin subcutaneously (Sandoz S.p.A., Milano, Italy). At the end of this period, 4 rats were killed with an overdose of ether. Groups of 4 of the remaining rats were killed at $10,20,40$, and 60 days.

A control group of 10 rats was injected subcutane- ously for 21 days with the same volume of the vehicle alone: 5 animals were killed after withdrawal of vehicle administration (control Group I) and 5 after 60 days (control Group 2). The mean weight gain of rats treated with calcitonin and controls was similar.

Radiographs of both tibiae were taken on Kodak mammographic film. The proximal epiphysis and metaphysis of each bone were dissected from soft tissues and fixed in neutral formalin for histological study. Evaluation of radiographs and histological slides was made blindly and independently by two of the authors (AN and UEP).

After decalcification in EDTA for 2 weeks, the specimens were embedded in paraffin and sections cut in the middle coronal plane of the metaphysis. Hematoxylin-eosin stained sections were evaluated for histomorphometry with a Leitz M 15 low-power microscope equipped with a grid ocular. The distance between 2 intersections of the grid was adjusted with a reference caliper resting on the coverslip; the measurement on the grid was then corrected according to this factor and expressed in $\mathrm{mm}$. The height of the perichondrial ring was measured in the middle coronal plane sections of the right and left metaphyses of each rat. Since the effect of arrest of metaphyseal modeling is present occasionally on both the medial and lateral sides of the metaphysis, but is a constant finding on the medial side, only the height of the former was measured. The right or left tibia of each rat was randomly used for evaluation and compared with control Group 1. Control Group 2 was matched against control Group 1 in 
order to check differences of perichondrial ring height determined by the rat's growth. In each group the left and right tibia were also compared.

Osteoclasts were counted with a Leitz Aristoplan microscope (enlargement $250 \times$ ) in the same coronal slide of the proximal tibia: the area utilized for the quantitative assessment included the whole epiphysis,

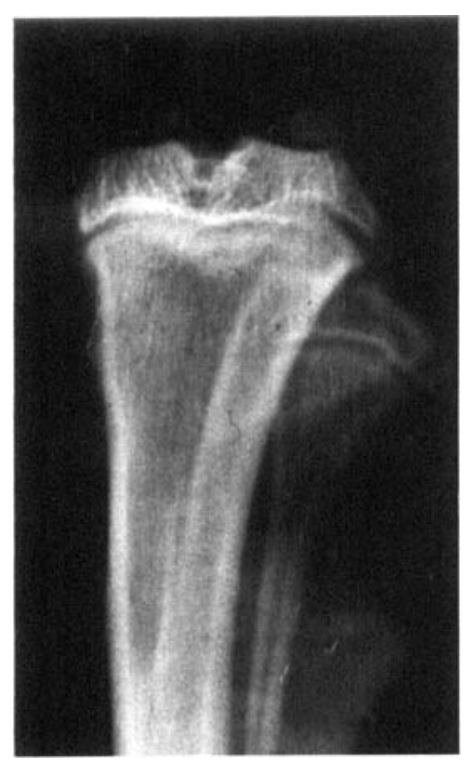

Figure 1. The proximal tibia in control rats. growth plate and metaphysis. The lower margin of this area was a line parallel to the growth plate and drawn to a distance from the top margin of the growth plate equal to twice the height of the epiphysis. The density of osteoclasts was expressed as the ratio between the number of cells and the surface area $\left(\right.$ cells $\left./ \mathrm{mm}^{2}\right)$. Only multinuclear cells closely associated with both trabecular and cortical endosteal surfaces were identified as osteoclasts.

The selection of groups for statistical evaluation was the same as for the perichondrial ring height assessment. The Student's $t$-test was used.

\section{Results}

Typical changes were present in all the rats after 21 days treatment. Radiographic thickening of the marginal growth plate on the medial side was evident (Figures 1 and 2). Histologically these zones were characterized by persistence of columns of hypertrophic chondrocytes (Figure 3). The corresponding metaphysis had a cylindrical shape due to arrest of modeling (failure of conization), and the perichondrial ring was consequently higher than normal (Table 1). The number of osteoclasts was increased, and their nuclei had a dense chromatin or were frankly pyknotic.

10 days after withdrawal of therapy, extension of growth plate cartilage into the metaphysis was no longer evident (Figure 2). In the corresponding histo-

Figure 2. The proximal tibia in rats treated with salmon calcitonin 100 units $/ \mathrm{kg} / \mathrm{day}$ for 21 days at time 0 (left), 10 days (center) and 60 days (right) after withdrawal of therapy.

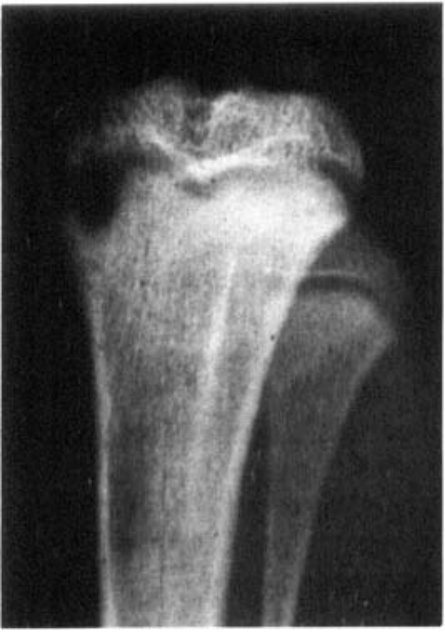

At time 0 , extension of the growth plate and arrest of metaphyseal modeling on the medial side are evident.

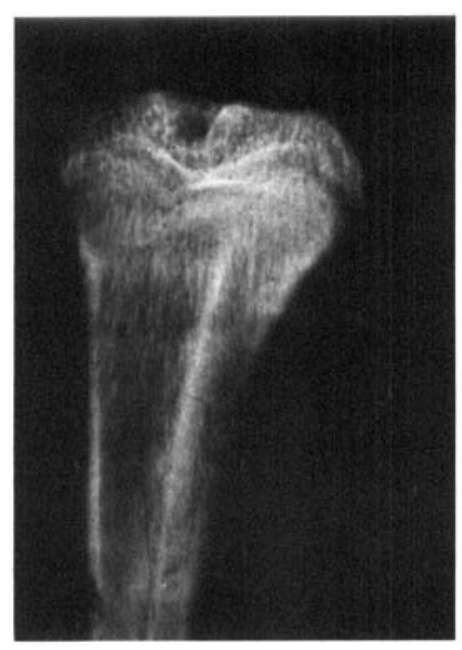

At 10 days, extension of the growth plate inside the metaphysis is no longer seen and renewed metaphyseal modeling is present. However, the medial margin is irregular and shaded.

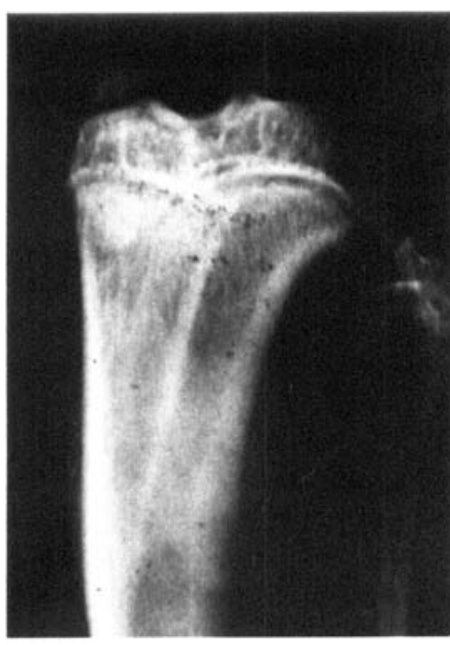

At 60 days, a denser opaque area is evident on the medial part of the metaphysis. A structural alteration suggesting a giant-cell-like tumor is present in the head of the fibula. 

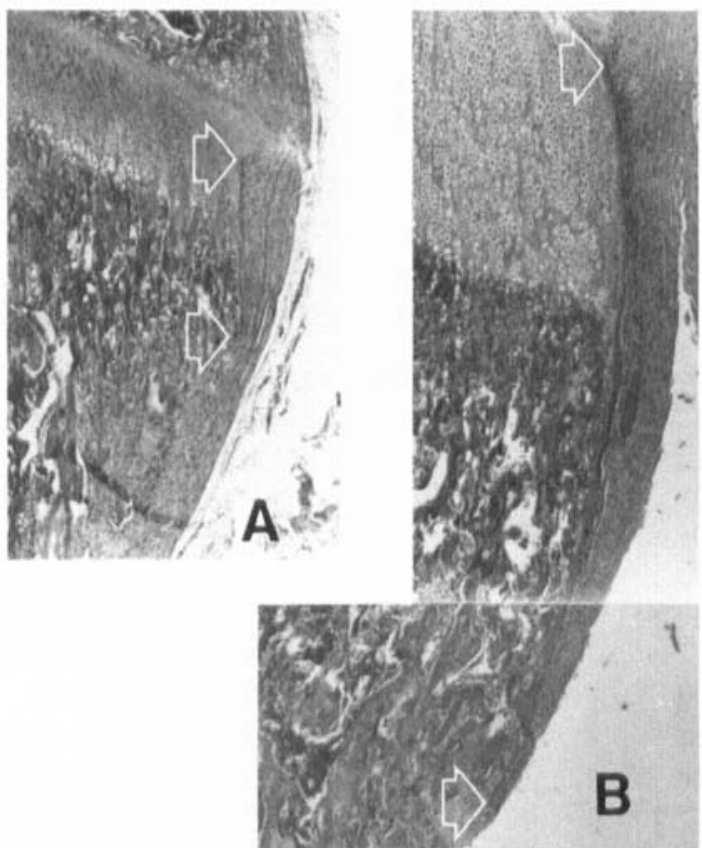

Figure 3. The medial growth plate cartilage and metaphysis in controls (A) and in rats after 21 days of treatment with salmon calcitonin (B). Arrows indicate the top and the bottom of the perichondrial ring. $\mathrm{HE}, \times 30$.

logical slides the abnormally high columns of hypertrophic chondrocytes had disappeared, the perichondrial ring was still higher than in the controls, but signs of renewed metaphyseal modeling were present at the bottom end of the ring, forming a notch on its inner aspect (Figure 4). In this area, the distribution of osteoclasts was very dense: multinuclear cells with a large, finely-granular cytoplasm and nuclei with sparse chromatin were present, together with cells with pyknotic nuclei and scanty cytoplasm (Figure 5).

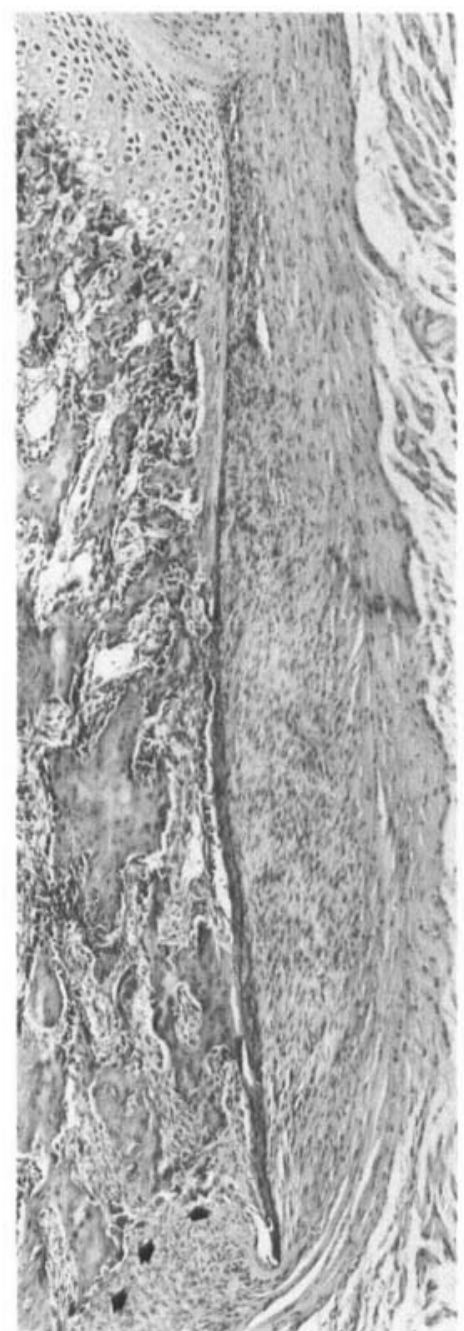

Figure 4. The perichondrial ring 10 days after withdrawal of calcitonin therapy is higher than in controls. However, renewed bone resorption is occurring at the bottom end of the ring (arrows). In the growth plate, the abnormally high columns of hypertrophic chondrocytes have disappeared. $\mathrm{HE}, \times 100$.

Table 1. Mean height $(\mathrm{mm} S D)$ of the medial perichondrial ring and number of osteoclasts $\left(\mathrm{m} / \mathrm{mm}^{2} \mathrm{SD}\right)$ in rat tibias. The rats had been treated with salmon calcitonin $100 \mathrm{U} / \mathrm{kg} /$ day for 21 days and then killed after $0-60$ days. The controls were killed after 0 or 60 days. The experimental groups and the 60 days control group were compared with 0 days control group using the Student's t-test

\begin{tabular}{|c|c|c|c|c|c|c|c|}
\hline \multirow{2}{*}{$\begin{array}{l}\text { Days after } \\
\text { treatment } \\
0\end{array}$} & \multirow{2}{*}{$\frac{n}{4}$} & \multicolumn{2}{|c|}{ Perichondrial ring } & \multirow{2}{*}{$\begin{array}{c}P \\
0.02\end{array}$} & \multicolumn{2}{|c|}{ Osteoclasts } & \multirow{2}{*}{$\begin{array}{c}P \\
<0.001\end{array}$} \\
\hline & & 3.47 & 0.59 & & 2.33 & 0.35 & \\
\hline 10 & 4 & 3.12 & 0.72 & $<0.02$ & 1.79 & 0.27 & $<0.02$ \\
\hline 20 & 4 & 1.56 & 0.66 & ns & 1.33 & 0.36 & ns \\
\hline 40 & 4 & 1.71 & 0.69 & ns & 1.21 & 0.36 & ns \\
\hline 60 & 4 & 1.02 & 0.49 & $<0.05$ & 1.16 & 0.20 & ns \\
\hline control 1 & 5 & 1.79 & 0.44 & ns & 1.32 & 0.19 & ns \\
\hline control 2 & 5 & 1.71 & 0.31 & ns & 1.30 & 0.18 & ns \\
\hline
\end{tabular}




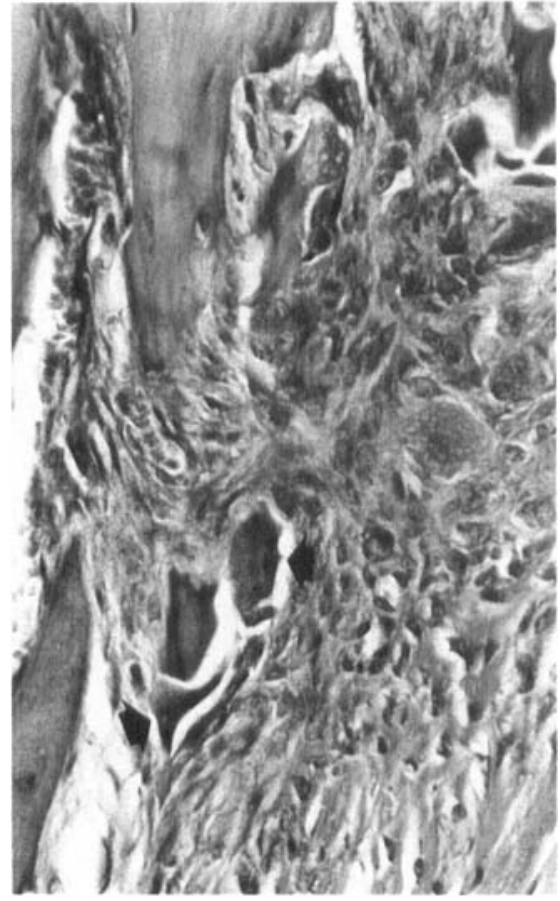

Figure 5. Detail of the area at the bottom end of perichondrial ring 10 days after withdrawal of calcitonin therapy: a large number of osteoclasts with abundant, finely-granukar cytoplasm are present; a few osteoclasts with denser cytoplasm and pyknotic nuclei are still evident (arrows). HE, $\times \mathbf{4 0 0}$

At later intervals, the height of the perichondrial ring progressively decreased, as well as the number of osteoclasts. In the groups studied 20 and 40 days after withdrawal of therapy, no difference in perichondrial ring height was present, while in rats killed after 60 days, the ring was even lower than in the controls. No differences were observed between control groups.

The mean number of osteoclasts was higher than in controls at 0 and 10 days. There were no differences from 20 days onward or between the control groups. Comparisons of perichondrial ring height and mean number of osteoclasts between the right and left proximal tibia in each group and in controls also did not differ.

The radiographic evolution of the area of cartilage extension showed a gradual healing with time. However, after 60 days, a denser, opaque area was evident on the medial aspect of the metaphyses. Failure of conization was corrected from 20 days onward.

\section{Discussion}

The height of the perichondrial bone ring is a reliable indicator of activity of the resorbing cells. Since it is formed by differentiated osteoblasts at the periphery of the growth plate and is resorbed by osteoclasts at the bottom, the constant height of the ring during growth can be maintained only if apposition at the top is equal to resorption at the bottom. Therefore, an increased height of the ring may be due to increased apposition or reduced resorption. In another study (Pazzaglia et al. 1993), no differences in the total tibial length between calcitonin-treated rats and controls were observed as would be expected in case of accelerated apposition. thus confirming that resorption was reduced in our calcitonin rats.

The observed persistence of hypertrophic chondrocytes in the growth plate of rats treated with high doses of salmon calcitonin has previously been reported (Burch and Corda 1985), but these findings have been interpreted as stimulation of chondrocytes rather than modeling inhibition; but again if such an interpretation is correct, an increased total length of the bone should be observed.

The arrest of modeling of the growth plate cartilage has been reported to be associated with failure of mineralization of intercolumnar septa and primary metaphyseal trabeculae (Pazzaglia et al. 1993). The same phenomenon is present with other inhibitors of bone remodeling, like diphosphonates (Schenk et al. 1973) or in human pathological conditions, like rickets or mucolipidosis 2 (Pazzaglia et al. 1989). The correlation between these two phenomena remains obscure, and no causal relationship has so far been documented. However, this association can explain the radiotransparent area on the medial aspect of the unmodeled metaphysis after 21 days of calcitonin administration; recovery of bone resorption activity after suspension of treatment is accompanied by renewed calcification, as shown by the radiographs at 10 days. Following the block of modeling, uncalcified primary metaphyseal trabeculae become denser and when recalcified they produce a more opaque area in the metaphysis, as noted at 60 days.

In conclusion, the resorption activity of osteoclasts appears to be closely associated with the calcification process. Both must function 10 days after withdrawal of high doses of calcitonin, even if the quantitative assessment of resorption activity (by perichondrial ring height measurement) became significant only after 20 days.

There is also a striking resemblance of the multinuclear cells at the distal end of the perichondrial bone bark and the outer aspect of the metaphysis of rats 
treated with high doses of calcitonin and those observed when diphosphonates are given (Schenk et al. 1973). Ultrastructural study of the large polycarios present in the latter suggests that these are osteoclasts which have lost the brush border and are no longer capable of resorbing bone (Miller et al. 1977). The conclusions drawn from the morphological changes in these cells are confirmed in the present study by the increased height of the perichondrial ring, which documents the arrest of the resorbing activity. A paradox becomes apparent where the number of osteoclasts increases, but the resorption activity is inhibited. A possible explanation rests on the fact that transient hypocalcemia, induced by high doses of calcitonin administration, produces secondary hyperparathyroidism (Glajchen et al. 1990) with stimulation of osteoclast formation (Baron and Vignery 1981). However, these cells are not capable of resorbing bone because of the inhibitory effect of calcitonin itself. Other factors may contribute to the increased number (Hedlund et al. 1983) of large polynucleated cells: their appearance is such that they are more easily detected in histological slides or the life span of these cells is increased. Whichever hypothesis is true, these osteoclasts are not active and no reliable assessment of bone resorption can be based on their number.

When calcitonin administration was interrupted, the recovery of resorption activity was shown with the morphometric analysis of periochondrial ring height after 20 days and it was accompanied by a reduction in the number of osteoclasts as well as by normalization of their appearance. A possible explanation of this aspect is that a new population of young osteoclasts is recruited, while the old inactive ones progressively disappear.

The effects we have observed were obtained with very high doses of the hormone, which are never used in clinical therapy.

\section{References}

Baron R, Vignery A. Behavior of osteoclasts during rapid change in their number induced by high doses of parathyroid hormone or calcitonin in intact rat. Metab Bone Dis Rel Res 1981: 2: 339-46.

Burch W M, Corda G. Calcitonin stimulates maturation of mammalian growth plate cartilage. Endocrinology 198.5; $116(5): 1724-8$

Chambers T J. McSheehy P M, Thomson B M. Fuller K. The effect of calcium-regulating hormones and prostaglandins on bone resorption by osteoclasts disaggregated from neonatal rabbit bones. Endocrinology 1985; 116 (1): 234-9.

Glajchen N, Thomas S, Jowell P, Epstein S, Ismail F, Fallon $M$. The effect of high-dose salmon calcitonin on bone mineral metabolism in the normal rat. Calcif Tissue Int 1990; 46 (1): $28-32$.

Hedlund T, Hulth A, Johnell O. Early effects of parathormone and calcitonin on the number of osteoclasts and on serum calcium in rats. Acta Orthop Scand 1983:54 (6): 802-4.

Miller S C, Jee W S, Kimmel D B, Woodbury L.. Ethane-1hydroxy-1, 1-diphosphonate (EHDP) effects on incorporation and accumulation of osteoclast nuclei. Calcif Tissue Res 1977; 22 (3): 243-52.

Pazzaglia U E, Beluffi $G$, Bianchi E, Castello A, Coci A, Marchi A. Study of the bone pathology in early mucolipidosis II (I-cell disease). Eur J Pediatr 1989; 148 (6): 553-7.

Pazzaglia U E, Zatti G, Di Nucci A, Coci A. Inhibitory effect of salmon calcitonin on bone resorption: morphological study of the tibial growth plate in rats. Calcif Tissue Int 1993; $52(2): 125-9$

Reynolds J J, Dingle J T. A sensitive in vitro method for studying the induction and inhibition of bone resorption. Calcif Tissue Res 1970: 4 (4): 339-49.

Russel R G, Kislig A M, Casey P A, Fleisch H, Thornton J Schenk R, Williams D A. Effect of diphosponates and calcitonin in the chemistry and quantitative histology of rat bones. Calsif Tissue Res 1973; 11 (3): 179-95.

Schenk R, Merz W A, Muhlbauer R, Russell R G, Fleisch H. Effect of ethane-1-hydroxy-1,1-diphosphonate (EHDP) and dichloromethylene diphosphonate ( $\mathrm{Cl} 2 \mathrm{MDP}$ ) on the calcification and resorption of cartilage and bone in the tibial epiphysis and metaphysis of rats. Calcif Tissue Res 1973; $11(3)$ : 196-214.

Stewart P J, Stern P H. Vertebral bone resorption in vitro: effects of parathyroid hormone calcitonin, 1,25-dihydroxy vitamin D3, epidermal growth factor, prostaglandin E2 and estrogen. Calcif Tissue Int 1987; 40: 21-6. 